\title{
Prevalence of Candida auris in Canadian acute care hospitals among at-risk patients, 2018
}

\author{
Hector Felipe Garcia-Jeldes', Robyn Mitchell², Allison McGeer ${ }^{3}$, Wallis Rudnick², Kanchana Amaratunga², \\ Snigdha Vallabhaneni ${ }^{4}$, Shawn R. Lockhart ${ }^{4}$, CNISP C. auris Interest Group and Amrita Bharat ${ }^{5^{*}}$ (D)
}

\begin{abstract}
To identify the prevalence of $C$. auris in Canadian patients who are potentially at risk for colonization, we screened 488 patients who were either hospitalized abroad, had a carbapenemase-producing organism (CPO), or were in units with high antifungal use. Two patients were colonized with C. auris; both had received healthcare in India and had a CPO. Among 35 patients who had recently received healthcare in the Indian subcontinent and were CPO colonized or infected, the prevalence of $C$. auris was $5.7 \%$.
\end{abstract}

Keywords: Candida, Cross infection, Drug resistance, Risk factors

\section{Background}

Candida auris is an emerging multidrug resistant pathogen associated with global hospital outbreaks [1]. Similar to other Candida species, the crude mortality rate of candidemia due to $C$. auris is $30-60 \%$ [2].

C. auris infection was first reported from Japan in 2009. Retrospective review of a large isolate collection identified an isolate from 1996, however, it appears that $C$. auris has very rarely caused human infection in the past [1]. Over the last decade, four distinct clades of $C$. auris have emerged and evolved independently, with frequent interhospital and inter-country transmission [2-5]. A single isolate belonging to a potential fifth clade was recently identified in Iran [6]. Whether the ongoing emergence of C. auris in different parts of the world will be mainly driven by the transmission of known clades, or whether new clades will continue to emerge remains uncertain.

There have been case reports of importation of $C$. auris by patients with recent exposure to healthcare in a

\footnotetext{
* Correspondence: Amrita.Bharat@canada.ca

${ }^{5}$ National Microbiology Laboratory, Public Health Agency of Canada, H5050 1015 Arlington St, Winnipeg, MB R3E 3R2, Canada

Full list of author information is available at the end of the article
}

country where $C$. auris is documented; these patients are often co-colonized with a carbapenemase producing organism (CPO) [7-10]. C. auris infection is often associated with underlying illnesses and outbreaks have been reported in intensive care units in multiple countries [11-13]. C. auris infection is also associated with previous exposure to antibiotics or antifungals $[14,15]$. Immunocompromised patient populations such as those in haematology/oncology wards or solid organ transplant wards often receive prophylactic antifungals [16, 17]; additionally, antifungal use was reported to be higher in ICUs compared to non-ICU wards [18]. Many of the risk factors for $C$. auris colonization are also risk factors for $\mathrm{CPO}$ colonization; these common risk factors include critical illness and comorbid conditions, prolonged hospitalization, and receipt of antimicrobials [19]. This may explain why patients who are colonized with $C$. auris are often co-colonized with a CPO.

As of March 2020, 24 cases of C. auris colonization and infection in Canada have been voluntarily reported to the Public Health Agency of Canada. The first case of multi-drug resistant $C$. auris was identified in Canada in 2017 in a patient who had recently received healthcare 
in the Indian subcontinent and was co-colonized with a carbapenemase-producing organism (CPO) [20]. C. auris is reportable in only one of 13 Canadian provinces and territories, and there is little data or recommendations to inform screening programs. We aimed to identify the prevalence of $C$. auris in Canada to inform national guidelines for screening and infection prevention and control.

\section{Methods}

The study included 23 acute care hospitals, of which 16 hospitals were participating in the Canadian Nosocomial Infection Surveillance Program (CNISP) and seven were Canadian Hospital Epidemiology Committee (CHEC) hospitals. We also included CPO colonized patients who were part of a prospective cohort study recruited from a 25 hospital research network, hereafter referred to as the "prospective cohort study" [21]. From September 4-November 6, 2018, hospitals screened patients in the following risk groups deemed by the study investigators to be potentially at risk for $C$. auris colonization: patients being admitted to study hospitals with recent hospitalization outside of Canada [Group 1]; patients being admitted to study hospitals with recent travel to the Indian subcontinent without hospitalization [Group 2]; CPO colonized or infected inpatients or outpatients [Group 3]; inpatients in hospital units that are associated with intensive antifungal use [Group 4]; and hospital contacts of a C. auris index case [Group 5] (Table 1). Patients who met more than one criteria were assigned to the first risk group identified. Patients in Groups 1 and 2 were identified through risk-based screening questions already in place in the hospitals. Patients in Group 3 included CPO-colonized inpatients and outpatients in the

Table 1 Eligibility criteria for patient risk groups that were screened for $C$. auris

\begin{tabular}{|c|c|c|}
\hline Group & Description & Notes \\
\hline $\begin{array}{l}\text { Group } \\
1(n= \\
92)\end{array}$ & $\begin{array}{l}\text { recent hospitalization outside } \\
\text { of Canada }\end{array}$ & $\begin{array}{l}\text { time periods defining recent } \\
\text { overseas hospitalization }(0.5, \\
1 \text {, or } 2 \text { years) varied as per } \\
\text { each hospital policy }\end{array}$ \\
\hline $\begin{array}{l}\text { Group } \\
2(n= \\
117)\end{array}$ & $\begin{array}{l}\text { recent travel to the Indian } \\
\text { subcontinent without } \\
\text { hospitalization }\end{array}$ & $\begin{array}{l}\text { time periods defining recent } \\
\text { travel ( } 1 \text { or } 2 \text { years) varied as } \\
\text { per each hospital policy }\end{array}$ \\
\hline $\begin{array}{l}\text { Group } \\
3(n= \\
104)\end{array}$ & $\begin{array}{l}\text { CPO colonized or infected } \\
\text { inpatients or outpatients and } \\
\text { consenting participants in a } \\
\text { different prospective CPO } \\
\text { cohort study }\end{array}$ & \\
\hline $\begin{array}{l}\text { Group } \\
4(n= \\
282)\end{array}$ & $\begin{array}{l}\text { units that are associated with } \\
\text { intensive antifungal use }\end{array}$ & $\begin{array}{l}\text { patients identified by hospital } \\
\text { census on a single day }\end{array}$ \\
\hline $\begin{array}{l}\text { Group } \\
5(n= \\
0)\end{array}$ & $\begin{array}{l}\text { hospital contacts of a C. auris } \\
\text { index case }\end{array}$ & $\begin{array}{l}\text { screening as per each } \\
\text { hospital policy }\end{array}$ \\
\hline
\end{tabular}

23 participating hospitals as well as consenting participants in the other prospective cohort study of CPO colonized patients who had visits in September or October of 2018. Group 4 patients were identified by hospital census on a single day during the study period in each participating hospital. Group 5 patients were identified through active surveillance in hospitals with $C$. auris cases. The time periods defining recent travel ( 1 or 2 years) and overseas hospitalization $(0.5,1$, or 2 years $)$ varied as per each hospital policy. Data were collected using a standardized data collection form that included demographic and potential $C$. auris risk factors.

Two Eswabs ${ }^{\text {Tx }}$ (Copan Diagnostics, Murietta, CA) were held together to obtain combined bilateral axilla/groin swabs. One tube was inoculated directly onto chromogenic agar, either CandiSelect $^{\bullet}$ (Bio-rad, 16 healthcare sites), Brilliance Candida (Thermo Scientific, 6 sites), or other non-selective media (1 site). The second tube was shipped to the US Centers for Disease Control and Prevention (CDC) for culture with broth pre-enrichment and chromogenic agar as previously described [22]. Broth pre-enrichment allows for preferential growth of C. auris over other Candida species by employing high salinity $(10 \% \mathrm{wt} / \mathrm{vol} \mathrm{NaCl})$, dulcitol as a carbon source, and incubation at elevated temperature $\left(40^{\circ} \mathrm{C}\right)$. Candida albicans colonies appear pink on CandiSelect ${ }^{\circ}$ or green on Brilliance Candida ${ }^{\circledR}$ chromogenic agar. C. auris was identified by screening non-albicans colonies identified on culture by MALDI-TOF (Bruker Biotyper or Vitek $\mathrm{MS}$ ). Isolates underwent whole genome sequencing on the NextSeq platform (Illumina, San Diego, CA) at the National Microbiology Laboratory Canada. Data were analyzed using Excel and Stata, v15 (StataCorp, Texas).

\section{Results}

The 23 participating acute care hospitals were located in six Canadian provinces: British Columbia $(n=7)$, Alberta $(n=2)$, Saskatchewan $(n=2)$, Ontario $(n=7)$, Quebec $(n=4)$, and New Brunswick $(n=1)$; the prospective cohort study for $\mathrm{CPO}$ colonization was taking place in south-central Ontario. During the study period, 488 at risk patients were screened: 45 in Group 1, 58 in Group 2, 103 in Group 3, 282 in Group 4, and none in Group 5. The median age of screened patients was 64 years (inter-quartile range (IQR) 42-74) and 253 (51.8\%) were male.

No C. auris isolates were identified among 282 patients from populations that are normally associated with higher antifungal use (Group 4) in Canada during the study period. This group included patients with haematologic malignancies or solid organ transplants ( $n=152$ patients), and patients admitted to intensive care units $(n=109)$ or oncology wards $(n=21)$. The median age of patients in Group 4 was 63 years and 56.5\% 
were male. In the 30 days prior to screening, 244 (89\%) of these patients had received an antibiotic and 85 (38\%) an antifungal; 167 (68\%) had a central venous catheter. Five of these patients reported travel outside North America without healthcare in the previous 12 months ( 3 to the Caribbean, 2 to East Asia, 1 to the United Kingdom) and one patient received healthcare abroad (in Central Africa).

There was considerable overlap in travel and healthcare associated risk factors in the 206 patients screened as part of Group 1 (hospitalization outside of Canada), Group 2 (travel to the Indian subcontinent without hospitalization) and Group 3 (CPO infection/ colonization) (Fig. 1). The median age of 92 patients who had healthcare outside of Canada was 68 years and $58.7 \%$ were male. The median time between overseas healthcare admission and screening in Canada was 5.8 months (interquartile range 2.8-9.6). Healthcare was received in India $(n=37,40 \%)$, the United States of America $(n=11,12 \%)$, Pakistan $(n=6,7 \%)$, China $(n=6,7 \%)$, Portugal $(n=3,3 \%)$, or other countries $(n=29,32 \%)$. Among 117 patients who travelled to the Indian

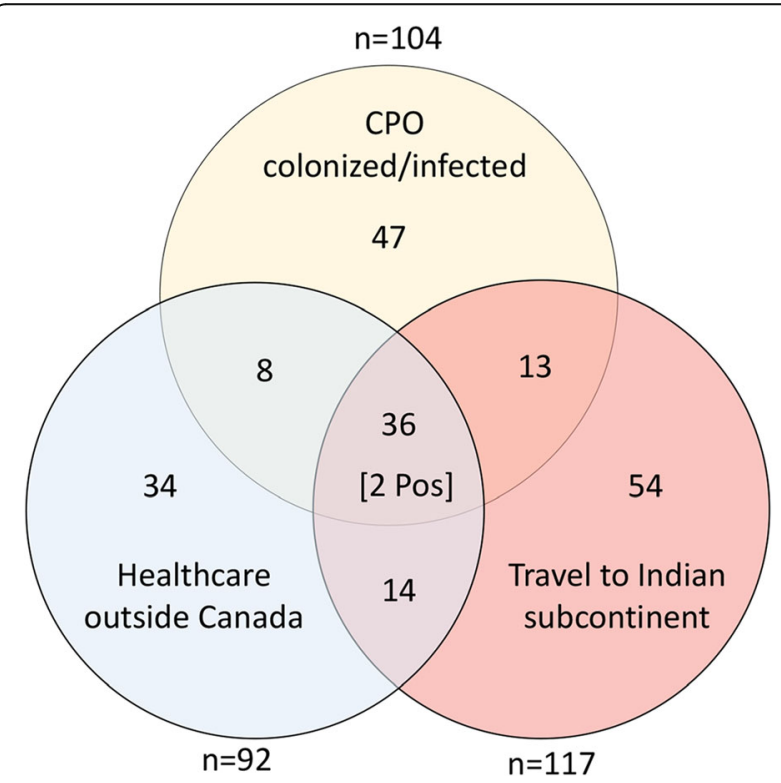

Fig. 1 Overlapping risk factors in 206 patients who had recent hospitalization outside Canada, recent travel to the Indian subcontinent, or previous/current CPO colonization. The number of instances of each risk factor is shown outside of each circle. Healthcare outside of Canada was received in India $(n=37)$, the United States of America $(n=11)$, Pakistan $(n=6)$, China $(n=6)$, Portugal $(n=3)$, or other countries $(n=29)$. CPO colonized patients carried NDM $(n=59)$, OXA-48 $(n=24)$, both NDM and OXA-48 $(n=9)$, KPC $(n=11)$, or VIM $(n=1)$. One third of patients in these three groups had more than one risk factor (71 of 206 patients). Of 36 patients who had all three risk factors, 35 received healthcare in the Indian subcontinent and one received healthcare in the Netherlands. The two patients who were positive for $C$. auris had recently travelled to India and received healthcare there, and were both co-colonized with NDM-1 and OXA-48-like carbapenemase-producing organisms subcontinent without hospitalization, the median age was 63 years and $43.6 \%$ were male. Among $104 \mathrm{CPO}$ colonized patients, the media age was 55 years and 51\% were male. CPO colonized patients carried NDM $(n=$ 59), OXA-48 $(n=24)$, both NDM and OXA-48 $(n=9)$, KPC $(n=11)$, or VIM $(n=1)$. Of 36 patients who had all three risk factors, 35 received healthcare in the Indian subcontinent and one received healthcare in the Netherlands. Two of these patients were found to be colonized with $C$. auris. C. auris was initially identified in both patients by MALDI-TOF and the results were confirmed by whole genome sequencing. Both $C$. auris colonized patients were hospitalized in India approximately 4 months prior to being screened for $C$. auris and both patients were known to be co-colonized with both NDM-1 and OXA-48-like producing organisms.

Thus, the overall prevalence of $C$. auris was $0.4 \%$ (2/488 patients; 95\% CI 0.1-1.5\%) in Canadian patients who were deemed to be at higher risk of fungal infections. Among the 35 patients in this study who had recently received healthcare in the Indian subcontinent and were CPO colonized or infected, the prevalence of $C$. auris colonization was $5.7 \%$ (95\% CI $0.7-19.2 \%$ ) compared to 0 of 453 without these two risk factors $(p=0.005)$.

One isolate was identified by both direct culture and broth pre-enrichment while the second isolate was identified only after a pre-enrichment for $C$. auris in broth. The second patient was undergoing daily chlorhexidine baths, which may have led to a lower burden of $C$. auris. A previous study found that direct culture resulted in detection of C. auris in $75 \%$ of the specimens that were positive by broth pre-enrichment [22]. At the time of $C$. auris detection, both patients were already under contact precautions due to their $\mathrm{CPO}$ colonization status; contact screening for $C$. auris was not carried out. Whole genome sequencing and phylogenetic analysis showed that both isolates belonged to South Asian Clade I, consistent with recent exposure to healthcare in India. In our study, the isolates were identified at the same healthcare facility, however they differed by 70 single nucleotide variants (SNVs), consistent with separate introductions into the facility. Within Clade 1, the US CDC previously found that genetically distinct isolates from multiple states differed by $62 \mathrm{SNVs}$ (range 41-88) whereas isolates in an epidemiologically-linked cluster differed by a median of three SNVs (range 0-12) [3].

\section{Discussion}

In this study, the overall prevalence of colonization with C. auris among Canadian patients at potential risk of colonization was low $(0.4 \%)$. C. auris was only identified in patients who were both colonized/infected with CPO and who had recently received inpatient healthcare in the Indian subcontinent. 
Our findings support the hypothesis that the greatest risk from $C$. auris is posed by spread of existing clades from endemic areas to new areas, as opposed to the emergence of new strains associated with increasing antifungal use. Importation of all four clades of $C$. auris into the United States from other countries has also been documented [3]. These data are consistent with the recommendation of the US CDC for admission screening of patients who have had an overnight hospitalization in the previous 12 months in a country where $C$. auris has been documented, especially if they also have a CPO [23]. However, our study did not evaluate all risk groups. For instance, our study did not screen residents of long term acute care facilities, who have been found to be a risk group in the United States [24]. Although $C$. auris colonization currently appears to be uncommon in Canadian hospitals, its rapid spread in other countries suggests that Canadian hospitals should consider active screening of high risk groups and contacts of index cases to permit early detection and limit the spread of C. auris [5].

\section{Acknowledgements}

We are grateful to the microbiology laboratory and infection prevention and control staff at participating hospitals for their contributions. We also thank the DNA Core Facility at the National Microbiology Laboratory for support with whole genome sequencing. All authors declare that there is no conflict of interest.

Other members of the CNISP C. auris interest group include Ghada AlRawahi, British Columbia Children's Hospital, BC Women's Hospital, Vancouver, BC; Elizabeth Brodkin, Fraser Health Authority, Vancouver, BC; Marthe Charles, Vancouver General Hospital, Vancouver, BC; Jeannette Comeau, IWK Health Centre, Halifax, NS; Ian Davis, QEll Health Sciences Centre, Halifax, NS; Johan Delport, Maisonneuve-Rosemont Hospital, Montreal, QC; Tanis C. Dingle, Alberta Public Laboratories, Edmonton, AB; Philippe Dufresne, Laboratoire de santé publique du Québec, Sainte-Anne-de-Bellevue, QC; Chelsey Ellis, The Moncton Hospital, Moncton, NB; Joanne Embree, Health Sciences Centre, Winnipeg, MB; Charles Frenette, McGill University Health Centre, Montreal, QC; George Golding, National Microbiology Laboratory, Public Health Agency of Canada, Winnipeg, MB; Linda M. Hoang, British Columbia Centre for Disease Control Public Health Laboratory, Vancouver, BC; Susy Hota, University Health Network, Toronto, ON; Kevin C. Katz, North York General Hospital, Toronto, ON; Pamela Kibsey, Royal Jubilee Hospital, Victoria, BC; Julianne V. Kus, Public Health Ontario, Toronto, ON; Joanne M. Langley, IWK Health Centre, Halifax, NS; Bonita E. Lee, Stollery Children's Hospital, Edmonton, AB; Marie-Astrid Lefebvre, Montreal Children's Hospital, Montreal, QC; Yves Longtin, SMBD-Jewish General Hospital, Montreal, QC; Kathy Malejczyk, Regina Qu'Appelle Health Region, Regina, SK; Shazia Masud, Surrey Memorial Hospital, Surrey, BC; Dominik Mertz, McMaster University and Hamilton Health Sciences, Hamilton, ON; Michael R. Mulvey, National Microbiology Laboratory, Public Health Agency of Canada, Winnipeg, MB; Susan Poutanen, Mount Sinai Hospital, Toronto, ON; Dale Purych, Surrey Memorial Hospital, Surrey, BC; Rajni Rantelidis, William Osler Health System, Brampton, ON; David Richardson, William Osler Health System, Brampton, ON; llan S. Schwartz, University of Alberta, Edmonton, AB; Stephanie Smith, University of Alberta Hospital, Edmonton, AB; Maxime-Antoine Tremblay, SMBD-Jewish General Hospital, Montreal, QC; Titus Wong, Vancouver General Hospital, Vancouver, BC; and Deborah Yamamura, Hamilton General Hospital, ON.

\section{Disclaimer}

The findings and conclusions in this report are those of the authors and do not necessarily represent the official position of the Centers for Disease Control and Prevention.

\section{Authors' contributions}

All authors contributed to the study design. SRL, AB, and the CNISP C. auris Interest Group contributed laboratory data. RM, WR, AB, AM, and SV contributed to data analysis and data interpretation. All authors contributed to writing and editing the manuscript. The authors read and approved the final manuscript.

\section{Authors' information}

Dr. Hector Felipe Garcia-Jeldes is a Medical Microbiologist at the CHU de Québec-Université Laval in Québec. His primary research interest is in reduction of healthcare associated infections.

\section{Funding}

This work was supported by internal funds from the Public Health Agency of Canada; the US Centers for Disease Control and Prevention; and the participating healthcare facilities.

\section{Availability of data and materials}

Sequence reads were deposited into the National Centre for Biotechnology Sequence Read Archive (Biosamples SAMN13424501 and SAMN13424502 in Bioproject PRJNA592373).

\section{Ethics approval and consent to participate}

At all participating CNISP and CHEC hospitals, this study was considered quality assurance with a waiver for ethics approval. Screening of the prospective cohort study patients was approved by the Sinai Health System Research Ethics Board.

\section{Consent for publication}

Not applicable.

\section{Competing interests}

The authors declare that they have no competing interests.

\section{Author details}

${ }^{1} \mathrm{CHU}$ de Québec-Université Laval, Québec, QC, Canada. ${ }^{2}$ Public Health Agency of Canada, Ottawa, ON, Canada. ${ }^{3}$ Mount Sinai Hospital, Toronto, ON, Canada. ${ }^{4}$ US Centers for Disease Control and Prevention, Atlanta, GA, USA. ${ }^{5}$ National Microbiology Laboratory, Public Health Agency of Canada, H5050 1015 Arlington St, Winnipeg, MB R3E 3R2, Canada.

Received: 30 March 2020 Accepted: 4 June 2020

Published online: 10 June 2020

\section{References}

1. Lockhart SR, Etienne KA, Vallabhaneni S, Farooqi J, Chowdhary A, Govender $\mathrm{NP}$, et al. Simultaneous emergence of multidrug-resistant Candida auris on 3 continents confirmed by whole-genome sequencing and epidemiological analyses. Clin Infect Dis. 2017;64:134-40.

2. Forsberg $\mathrm{K}$, Woodworth $\mathrm{K}$, Walters M, Berkow EL, Jackson B, Chiller T, et al. Candida auris: the recent emergence of a multidrug-resistant fungal pathogen. Med Mycol. 2019;57:1-12.

3. Chow NA, Gade L, Tsay SV, Forsberg K, Greenko JA, Southwick KL, et al. Multiple introductions and subsequent transmission of multidrug-resistant Candida auris in the USA: a molecular epidemiological survey. Lancet Infect Dis. 2018;18:1377-84.

4. Rhodes J, Abdolrasouli A, Farrer RA, Cuomo CA, Aanensen DM, ArmstrongJames D, et al. Genomic epidemiology of the UK outbreak of the emerging human fungal pathogen Candida auris. Emerg Microbes Infect. 2018;7:43.

5. Adams E, Quinn M, Tsay S, Poirot E, Chaturvedi S, Southwick K, et al. Candida auris in healthcare facilities, New York, USA, 2013-2017. Emerg Infect Dis. 2018;24:1816-24.

6. Chow NA, de Groot T, Badali H, Abastabar M, Chiller TM, Meis JF. Potential fifth clade of Candida auris, Iran, 2018. Emerg Infect Dis. 2019;25:e10062901781.

7. Brooks RB, Walters M, Forsberg K, Vaeth E, Woodworth K, Vallabhaneni S. Candida auris in a U.S. patient with Carbapenemase-producing organisms and recent hospitalization in Kenya. Morb Mortal Wkly Rep. 2019;68:664-6.

8. Hamprecht A, Barber AE, Mellinghoff SC, Thelen P, Walther G, Yu Y, et al. Candida auris in Germany and previous exposure to foreign healthcare. Emerg Infect Dis. 2019;25:41-1765. 
9. Vogelzang EH, Weersink AJL, van Mansfeld R, Chow NA, Meis JF, van Dijk K. The First Two Cases of Candida auris in The Netherlands. J Fungi (Basel). 2019:5:91.

10. Khan A, Shropshire WC, Hanson B, Dinh AQ, Wanger A, Ostrosky-Zeichner L, et al. Simultaneous infection with Enterobacteriaceae and Pseudomonas aeruginosa harboring multiple Carbapenemases in a returning traveler colonized with Candida auris. Antimicrob Agents Chemother. 2020;64:4.

11. Ruiz-Gaitán A, Martínez H, Moret AM, Calabuig E, Tasias M, Alastruey-lzquierdo $A$, et al. Detection and treatment of Candida auris in an outbreak situation: risk factors for developing colonization and candidemia by this new species in critically ill patients. Expert Rev Anti-Infect Ther. 2019;17:295-305.

12. Eyre DW, Sheppard AE, Madder H, Moir I, Moroney R, Quan TP, et al. A Candida auris outbreak and its control in an intensive care setting. N Engl J Med. 2018:379:1322-31.

13. Chakrabarti A, Sood P, Rudramurthy SM, Chen S, Kaur H, Capoor M, et al. Incidence, characteristics and outcome of ICU-acquired candidemia in India. Intensive Care Med. 2015;41:285-95.

14. Rudramurthy SM, Chakrabarti A, Paul RA, Sood P, Kaur H, Capoor MR, et al. Candida auris candidaemia in Indian ICUs: analysis of risk factors. J Antimicrob Chemother. 2017;72:1794-801.

15. Taori SK, Khonyongwa K, Hayden I, Athukorala GDA, Letters A, Fife A, et al. Candida auris outbreak: mortality, interventions and cost of sustaining control. J Inf Secur. 2019;79:601-11.

16. Pappas PG, Kauffman CA, Andes DR, Clancy CJ, Marr KA, Ostrosky-Zeichner L, et al. Clinical Practice Guideline for the Management of Candidiasis: 2016 Update by the Infectious Diseases Society of America. Clin Infect Dis. 2016; 62:e1-50.

17. Brizendine KD, Vishin S, Baddley JW. Antifungal prophylaxis in solid organ transplant recipients. Expert Rev Anti-Infect Ther. 2011;9:571-81.

18. Vallabhaneni S, Baggs J, Tsay S, Srinivasan AR, Jernigan JA, Jackson BR. Trends in antifungal use in US hospitals, 2006-12. J Antimicrob Chemother. 2018;73:2867-75.

19. Logan LK, Weinstein RA. The epidemiology of Carbapenem-resistant Enterobacteriaceae: the impact and evolution of a global menace. J Infect Dis. 2017:215:S28-36.

20. Schwartz IS, Hammond GW. First reported case of multidrug-resistant Candida auris in Canada. Can Commun Dis Rep. 2017:43:150-3.

21. Kohler PP, Melano RG, Patel SN, Shafinaz S, Faheem A, Coleman BL, et al. Emergence of Carbapenemase-producing Enterobacteriaceae, south-Central Ontario, Canada. Emerg Infect Dis. 2018;24:1674-82.

22. Welsh RM, Bentz ML, Shams A, Houston H, Lyons A, Rose LJ, et al. Survival, persistence, and isolation of the emerging multidrug-resistant pathogenic yeast Candida auris on a plastic health care surface. J Clin Microbiol. 2017; 55:2996-3005.

23. Centers for Disease Control and Prevention. Screening for Candida auris Colonization. 2018. www.cdc.gov/fungal/candida-auris/c-auris-screening. html. Accessed 27 Apr 2020.

24. Kerins JL, Tang AS, Forsberg K, Jegede O, Ealy M, Pacilli M, et al. 923. Rapid emergence of Candida auris in the Chicago region. Open Forum Infect Dis. 2018:5:528.

\section{Publisher's Note}

Springer Nature remains neutral with regard to jurisdictional claims in published maps and institutional affiliations.

Ready to submit your research? Choose BMC and benefit from:

- fast, convenient online submission

- thorough peer review by experienced researchers in your field

- rapid publication on acceptance

- support for research data, including large and complex data types

- gold Open Access which fosters wider collaboration and increased citations

- maximum visibility for your research: over $100 \mathrm{M}$ website views per year

At $\mathrm{BMC}$, research is always in progress.

Learn more biomedcentral.com/submissions 